\title{
Research on the Method of Solving Kinematics Parameters of Three-axis Dynamic Centrifuge
}

\section{Bang-Zhao Zhou (D1549084091@qq.com)}

Academy of Engineering Physics Institute of Systems Engineering https://orcid.org/0000-0001-5864486X

\section{Shuai Zhang}

Academy of Engineering Physics Institute of Systems Engineering

\section{Min Yang}

Academy of Engineering Physics Institute of Systems Engineering

\section{Sheng-Lai Chen}

Academy of Engineering Physics Institute of Systems Engineering

\section{Feng Ou}

Academy of Engineering Physics Institute of Systems Engineering Ya-Hong Zhang

Xian Jiaotong University: Xi'an Jiaotong University

\section{Original Article}

Keywords: Dynamic centrifugal test technology , Inverse kinematics , Time step adaptation

Posted Date: July 26th, 2021

DOl: https://doi.org/10.21203/rs.3.rs-730044/v1

License: (c) (i) This work is licensed under a Creative Commons Attribution 4.0 International License.

Read Full License 


\section{Title page}

\section{Research on the Method of Solving Kinematics Parameters of Three-axis Dynamic Centrifuge}

Bang-Zhao Zhou, born in 1992, is currently an engineer at Institute of Systems Engineering, China Academy of Engineering Physics, Mianyang, China. He received his PhD degree from Shanghai Jiaotong University, China, in 2020. His research interests include dynamic centrifugal test technology.

Tel: +86-15528551521; E-mail: $1549084091 @ q q . c o m$

Shuai Zhang, is currently an engineer at Institute of Systems Engineering, China Academy of Engineering Physics, Mianyang, China. E-mail: zhangshuai5994@126.com

Min Yang, is currently a Senior engineer at Institute of Systems Engineering, China Academy of Engineering Physics, Mianyang, China. E-mail: yang77.5@163.com

Sheng-Lai Chen, is currently a Senior engineer at Institute of Systems Engineering, China Academy of Engineering Physics, Mianyang, China.

E-mail: chensl@caep.cn

Feng Ou, is currently a Senior engineer at Institute of Systems Engineering, China Academy of Engineering Physics, Mianyang, China. E-mail: oufeng_oo@163.com

Ya-Hong Zhang, is currently an associate professor at School of Aeronautics and Astronautics, Xi'an Jiaotong University, Xi'an, China. E-mail: zyhxjtu@xjtu.edu.cn

\section{Corresponding author: Bang-Zhao Zhou E-mail: 1549084091@qq.com}




\title{
Research on the Method of Solving Kinematics Parameters of Three-axis Dynamic Centrifuge
}

\author{
Bang-Zhao Zhou ${ }^{1} \cdot$ Shuai Zhang ${ }^{1} \cdot$ Min Yang $^{1} \cdot$ Sheng-Lai Chen ${ }^{1} \cdot$ Feng Ou ${ }^{1} \cdot$ Ya-Hong Zhang $^{2}$
}

Received June xx, 202x; revised February xx, 202x; accepted March xx, 202x

(c) Chinese Mechanical Engineering Society and Springer-Verlag Berlin Heidelberg 2017

\begin{abstract}
With the development of aerospace technology, the maneuverability of various types of aircraft continues to improve, and these aircraft experience an overload environment with high acceleration and high acceleration rate. Due to the special influence brought by the high acceleration rate, the dynamic centrifugal test technology, which is different from the traditional steady-state centrifugal test technology, came into being. The steady-state centrifuge has only one rotor, while the dynamic centrifuge has multiple rotors; so, the relationship between the acceleration of the control point and the motion parameters of the rotors is more complicated. Therefore, a key issue of the dynamic centrifugal test technology is the inverse kinematics of the dynamic centrifuge, which is to calculate the kinematic parameters of the dynamic centrifuge according to the expected acceleration environment that needs to be simulated on the centrifuge. After the kinematic parameters is calculated, the control target of each rotor of the dynamic centrifuge could be known, then the expected acceleration environment could be produced. In this paper, 1) on the basis of the predecessors, the equation for solving the angular velocity of the main arm of the centrifuge is improved; 2) and then a time step adaptive method is proposed, which takes into account the calculation accuracy and efficiency. As a result, an inverse kinematics algorithm that is more accurate and adaptable to various acceleration history curve is obtained. Finally, the inverse kinematics algorithm in this paper is verified through experiments and numerical simulations.
\end{abstract}

Keywords: Dynamic centrifugal test technology $\bullet$ Inverse kinematics $\bullet$ Time step adaptation

Bang-Zhao Zhou

1549084091@qq.com

1 Institute of Systems Engineering, China Academy of Engineering Physics, Mianyang 621900, China

2 School of Aeronautics and Astronautics, Xi'an Jiaotong University, Xi'an 710049, China

\section{INTRODUCTION}

With the development of aerospace technology, the maneuverability of many aircraft continues to improve [1]. These aircraft experience an overload environment with high acceleration and high acceleration rate [2-6]. This high acceleration rate will affect the performance of the electronic components on the aircraft, and change the dynamic characteristics of the lightweight and thin plate shell structure on the aircraft [7-10]. At the same time, it also places higher requirements on the pilot's anti-overload capability [11-13]. Therefore, the dynamic centrifugal test technology, which is different from the traditional steady-state centrifugal test technology [14-19], came into being.

The steady-state centrifuge has only one rotor. In the steady-state centrifugal test, it is stipulated that the loading and unloading rate of the centrifuge must be slow enough. Therefore, the tangential acceleration is ignored in the steady-state centrifugal test, and the test acceleration $a$ is considered to be $R \omega_{1}{ }^{2}$. According to the test acceleration $a$ and the centrifuge arm length $R$, the angular velocity of the centrifuge can be quickly obtained as $\omega=\sqrt{a / R}$. For the dynamic centrifugal test [20], 1) in order to generate an overload environment with a high rate of change, the loading rate of the dynamic centrifuge may be very large, and 2) the dynamic centrifuge has multiple rotors (e.g., the GLJ-3R dynamic centrifuge shown in Fig. 1 has three rotors: main arm, outer frame and inner frame). Therefore, on the one hand, the axial and tangential acceleration must be both considered, and on the other hand, the coordinate transformation method of multi-body dynamics needs to be 
applied to obtain the acceleration at the control points on the dynamic centrifuge. As a result, the forward kinematics of the dynamic centrifuge is far more complicated than that of the steady-state centrifuge, and ultimately leads to the inverse kinematics of the dynamic centrifuge (that is, calculating the kinematic parameters of the dynamic centrifuge according to the size of the centrifuge and the expected acceleration environment that needs to be simulated on the centrifuge) is extremely complicated. Only by solving the inverse kinematics problem of the dynamic centrifuge can the control target of each rotor of the dynamic centrifuge be obtained, and then the expected acceleration environment could be generated. Therefore, the inverse kinematics problem of the dynamic centrifuge has received many attentions.

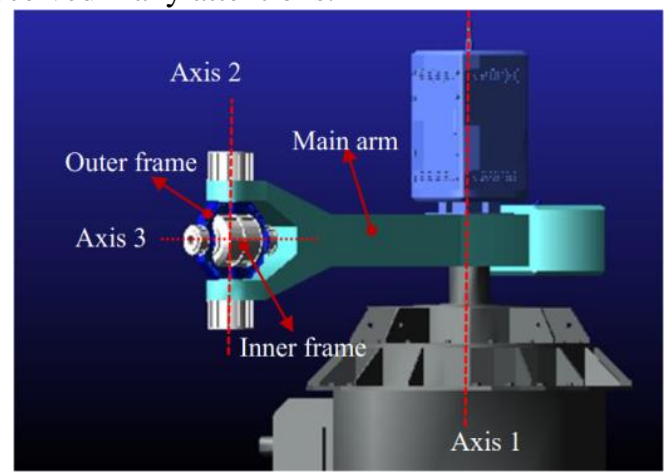

Fig. 1 GLJ-3R dynamic centrifuge of China Academy of Engineering Physics

In [21], a new type of three-channel centrifuge is proposed, and the analytical solution of the kinematic parameters of centrifuge the under the condition of uniform rotation is obtained. In [22], the biaxial acceleration based on the simplified kinematics model is obtained. In [23], the angular velocity of the main arm during the loading phase is solved. However, the solution in the unloading phase is more difficult. To solve this problem, in [24] Jacobi elliptic function is introduced to solve it, but the error of this method is a little large, reaching $0.49 \mathrm{~g}$ ( $\mathrm{g}$ is the acceleration of gravity). In [25], the two-dimensional interpolation is used to estimate the motion parameters of the unloading section, but it is necessary to establish a database before using this method. In [2], the method of constructing symmetrical loads is presented to solve this problem, the calculation accuracy is high, and the process is simple. However, the size of the time step has a great influence on the accuracy of the method, especially during the acceleration platform stage (that is, the stage where the acceleration is constant). In this paper, the equation for solving the angular velocity of the main arm of the centrifuge is derived again, and then a method which automatically selects the size of time step according to the accuracy is proposed for solving the equation, so both the calculation accuracy and efficiency are took into account in this paper. Finally, the algorithm in this paper is verified through experiments and numerical simulations, and the results shows that our inverse kinematics algorithm is accurate and adaptive to various kinds of acceleration history curve.

The contents of this paper are organized as follows. The forward and especially inverse kinematics of GLJ-3R dynamic centrifuge is introduced in Section 2; then the experiment and numerical simulations are shown in Section 3 to verify and determine the accuracy of our inverse kinematics algorithm; at last, the conclusion is in Section 4.

\section{THEORY}

\subsection{Forward Kinematics of GLJ-3R Dynamic Centrifuge}

The GLJ-3R dynamic centrifuge in Fig. 1 contains three rotors: main arm, outer frame and inner frame, and its schematic diagram can be simplified as shown in Fig. 2. The three rotors rotate around the Axes 1, 2, and 3 respectively. The rotation angle of the main arm relative to the ground is $q_{1}$, the rotation angle of the outer frame relative to the main arm is $q_{2}$, and the rotation angle of the inner frame relative to the outer frame is $q_{3}$; the coordinate system $O_{0}-x_{0} y_{0} z_{0}$ is an inertial system, $O-x_{1} y_{1} z_{1}$ is the body fixed frame of the main arm, $O-x_{2} y_{2} z_{2}$ is the body fixed frame of the outer frame, and $O-x_{3} y_{3} z_{3}$ is the body fixed frame of the inner frame. This article stipulates that when $q_{1}$ is zero, $x_{1}$ and $x_{0}$ coincide; when $q_{2}$ and $q_{3}$ are both zero, $O-x_{1} y_{1} z_{1}, O-x_{2} y_{2} z_{2}$ and $O-x_{3} y_{3} z_{3}$ coincide.

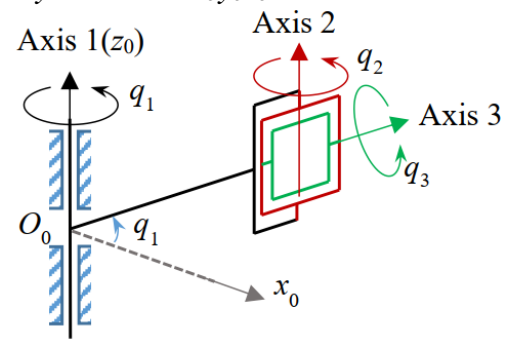

(a) schematic diagram of GLJ-3R dynamic centrifuge

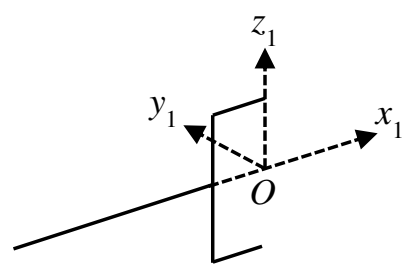

(b) main arm and its body-fixed frame 


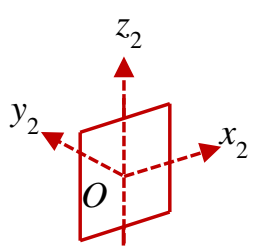

(c) outer frame and its body-fixed frame

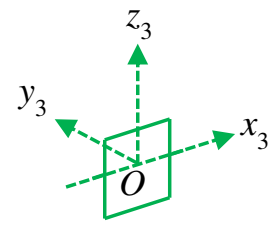

(d) inner frame and its body-fixed frame

Fig. 2 Schematic diagram of GLJ-3R dynamic centrifuge and its body-fixed frame

The point $O$ is fixed to the main arm, so the acceleration $\boldsymbol{a}$ of $O$ can be calculated according to the angular velocity and acceleration as

$$
\boldsymbol{a}^{(1)}=\left[\begin{array}{lll}
-R \& \mathbb{R} & R
\end{array}\right]^{\mathrm{T}}
$$

where $\boldsymbol{a}^{(1)}$ represents the coordinates of $\boldsymbol{a}$ in $O-x_{1} y_{1} z_{1}$, $R$ represents the length of $O_{0} O$, the superscript " $\mathrm{T}$ " represents matrix transpose. The coordinates of $\boldsymbol{a}$ in $O-x_{3} y_{3} z_{3}$ can be obtained through coordinate transformation as

$$
\boldsymbol{a}^{(3)}=\left[\begin{array}{l}
-R \& \cos q_{2}+R \sin q_{2} \\
\left(R \& \cos q_{2}+R \& \sin q_{2}\right) \cos q_{3} \\
-\left(R \& \cos q_{2}+R \& \sin q_{2}\right) \sin q_{3}
\end{array}\right]
$$

In the dynamic centrifugal test, the test object is fixed at point $O$ on the inner frame, so the acceleration of the object in its body-fixed frame $O-x_{3} y_{3} z_{3}$ is exactly $\boldsymbol{a}^{(3)}$. Therefore, the control objective of the dynamic centrifugal test is to ensure that $\boldsymbol{a}^{(3)}$ is equal to the expected acceleration of the test object. From Eq. (2), it can be seen that $\boldsymbol{a}^{(3)}$ is completely determined by the parameters $R$, \&, $q_{2}$, and $q_{3}$; here $R$ is a constant. The goal of this paper is to calculate the kinematic parameters \&, $q_{2}$, and $q_{3}$ of the dynamic centrifuge according to the expected acceleration $\boldsymbol{a}^{(3)}$ of the object.

\subsection{Inverse Kinematics of GLJ-3R Dynamic Centrifuge}

Calculating the kinematic parameters \&, $q_{2}$, and $q_{3}$ of the dynamic centrifuge according to the expected acceleration $\boldsymbol{a}^{(3)}$ of the object is an inverse kinematics problem. There are some nonlinear terms (e.g., \& , $\sin q_{2}$, $\sin q_{3}$ ) and the first two-order derivatives of $q_{1}$ in Eq. (2), so it is rather difficult to solve this equation directly. In order to simplify the solving, the magnitude $a$ of the expected acceleration $\boldsymbol{a}$ is firstly analyzed.

$$
a=R \sqrt{\& \neq+1}
$$

It can be seen that $a$ is completely determined by the kinematic parameter $q_{1}$ of the main arm. According to Eq. (3), $q_{1}$ can be calculated firstly, so as to avoid the direct solving of Eq. (2). Eq. (3) is a high-order nonlinear differential equation about $q_{1}$, which is not easy to solve directly. The following solution is based on the idea of the literature [2]. A small time period $\Delta t$ at the current moment $t$ is firstly took. Assuming that changes linearly during this period, then the \& at the next moment can be calculated as

$$
\$(t+\Delta t)=\&(t)+0.5[t(t)+\Delta t)] \Delta t
$$

In [2], \& $(t+\Delta t)$ is calculated by the rectangle integration formula, while the trapezoidal integration formula is used in this paper to refine the accuracy. The following equation can be obtained by substituting Eq. (4) into Eq. (3).

$$
\begin{aligned}
& C_{1}(t+\Delta t)+C_{2}(t+\Delta t)+C_{3}(t+\Delta t) \\
& +C_{4}(t+\Delta t)+C_{5}=0
\end{aligned}
$$

where $C_{1}=\Delta t^{4} / 16, \quad C_{2}=f_{q 1} \Delta t^{3} / 2, \quad C_{4}=2 f_{q 1}^{3} \Delta t$, $C_{3}=3 f_{q 1}{ }^{2} \Delta t^{2} / 2+1, \quad C_{5}=f_{q 1}{ }^{4}-[a(t+\Delta t) / R]^{2}, \quad$ and $f_{q 1}=\&(t)+\Delta t / 2$. It can be seen that Eq. (5) is a unary quartic equation about $(t+\Delta t)$. In the similar process of obtaining Eq. (5) in [2], the terms $C_{2}(t+\Delta t)$ and $C_{1}(t+\Delta t)$ are omitted since they contain $\Delta t^{3}$ and $\Delta t^{4}$ respectively. While in this paper, these two terms are both considered to refine the accuracy of Eq. (5). Besides, it is not too difficult to solve Eq. (5) even if these two terms are both considered, since this equation can be solved by the general solution formula of unary quartic equation. It is worth noting that in the unloading phase (that is, the phase when the acceleration magnitude $a$ drops), there may be a situation where Eq. (5) has no real roots. To solve this problem, the method of constructing symmetrical loads is presented in [2] to solve the problem, the calculation accuracy is high, and the process is simple. However, the size of the time step has a great influence on the accuracy of the method, especially during the acceleration platform stage (that is, the stage where the acceleration is constant). In this paper, a method which automatically selects the size of time step according to the accuracy is proposed for solving the Eq. (5), so both the calculation accuracy and efficiency are took into account in this paper.

\subsubsection{Method of adaptively selecting the size of time step}


It can be known from Eq. (3) that $= \pm \sqrt{a^{2} / R^{2}-t_{1}^{4}}$. The acceleration history curve in Fig. 3 is considered here, where $a$ increases before $t_{1}$ and keep constant after $t_{1}$. If $\&>0$, since $a$ increases before $t_{1}, \&$ also keeps increasing during this stage. Therefore, $\left(t_{1}\right)$ should be $+\sqrt{a^{2} / R^{2}-\&}$ since this solution is more than zero. Then at the moment of $t_{1}+\Delta t$, if $\left(t_{1}+\Delta t\right)=-\left(t_{1}\right)$, it can be obtained from Eq. (4) that $\&\left(t_{1}+\Delta t\right)=\$\left(t_{1}\right)$. Then it can be obtained that $a\left(t_{1}+\Delta t\right)=R \sqrt{\frac{q_{1}}{\left(t_{1}\right)+\left(t_{1}\right)}}=a\left(t_{1}\right)$ by substituting the $\left(t_{1}+\Delta t\right)$ and $\&\left(t_{1}+\Delta t\right)$ into Eq. (3). Therefore, $\left(t_{1}+\Delta t\right)=-\left(t_{1}\right)$ is the solution of at the moment of $t_{1}+\Delta t$. Similarly, at each subsequent time, the solution will be as follows: $\left(t_{1}+2 \Delta t\right)=\left(t_{1}\right)$, $\left(t_{1}+3 \Delta t\right)=-\left(t_{1}\right),\left(t_{1}+4 \Delta t\right)=\left(t_{1}\right) \ldots$ That is, oscillates at a high frequency of $1 / 2 \Delta t$ during the acceleration platform stage (this will be shown in Section 0 ). In fact, during the acceleration platform stage, the angular velocity of the main arm will be constant to ensure that the acceleration keeps unchanged; therefore, during the acceleration platform stage, will tend to $0, \&$ and will tend to $\sqrt{a / R}$. Therefore, this high-frequency oscillation solution is a defect of the above algorithm. When using the algorithm above to calculate the control parameters of the dynamic centrifuge, the algorithm should be improved. The improved program flow chart is shown in Fig. 4.

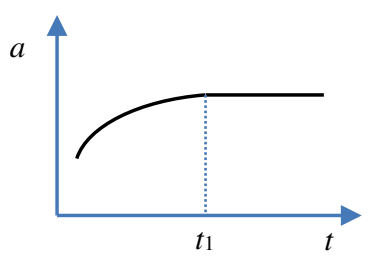

Fig. 3 History curve of the magnitude of acceleration

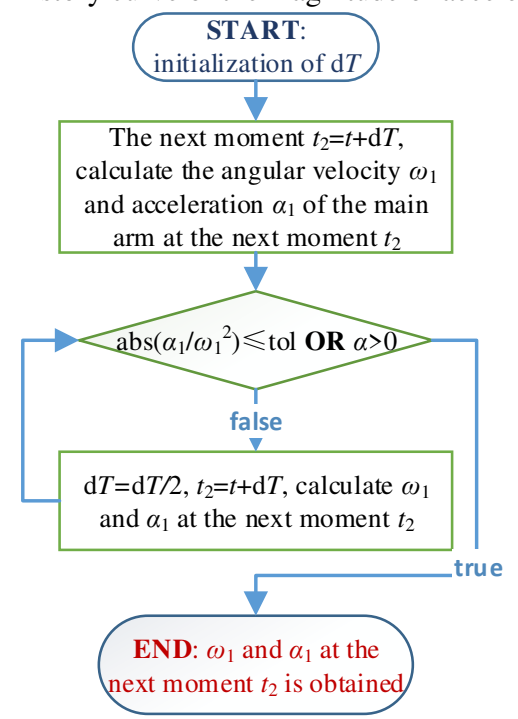

Fig. 4 Program flow chart for adaptively selecting the size of time step
In the case of acceleration history as shown in Fig. 3, $\omega_{1}$ has not reached its stable value during the acceleration platform stage at time $t_{1}$, and there is still room for its rise, so $\alpha_{1}>0$ is a true solution, here $\omega_{1}=\&$ and $\alpha_{1}=$. The tangential and normal accelerations of point $O$ on the main arm are $R \alpha_{1}$ and $-R \omega_{1}^{2}$ respectively, so abs $\left(\alpha_{1} / \omega_{1}^{2}\right)$ represents the relative magnitude of the tangential acceleration to the normal acceleration, and the $\operatorname{abs}\left(\alpha_{1} / \omega_{1}{ }^{2}\right)$ $\leqslant$ tol in the program means that within a certain accuracy range, $\alpha_{1}=0$. At this moment, since angular acceleration is nearly zero, the angular velocity of the main arm has nearly reached the stable value during the acceleration platform stage. Therefore, it is stipulated in this program that as long as abs $\left(\alpha_{1} / \omega_{1}^{2}\right) \leqslant$ tol or $\alpha_{1}>0$, the calculation will end, otherwise the time step $\mathrm{d} T$ will continue to be reduced.

\subsubsection{Rotation angle of the inner and outer frame}

After at the next moment is solved according to Eq. (5), then \& can be obtained from Eq. (4). Now, the unknown variables in Eq. (2) are $q_{2}$ and $q_{3}$, so $q_{2}$ and $q_{3}$ at the next moment can be solved according to Eq. (2) as

$$
q_{2}= \begin{cases}\arcsin \frac{a_{x}^{(3)}}{a}-\alpha, & (a \neq 0) \\ q_{2,0} & ,(a=0)\end{cases}
$$

where $a_{x}^{(3)}, a_{y}^{(3)}$, and $a_{z}^{(3)}$ are the three elements of $\boldsymbol{a}^{(3)}, q_{2,0}$ is the $q_{2}$ at the current moment, and $\alpha$ is

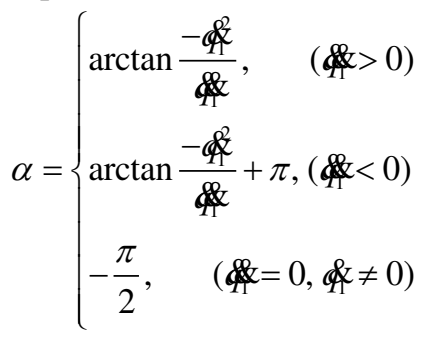

Then the $q_{3}$ at the next moment can be solved according to Eq. (2) as

$$
q_{3}=\left\{\begin{array}{lr}
\beta, & \left(\left|b \cos (\beta+\pi)-a_{y}^{(3)}\right|>\left|b \cos \beta-a_{y}^{(3)}\right|, a_{y}^{(3)} \neq 0\right) \\
\beta+\pi,\left(\left|b \cos (\beta+\pi)-a_{y}^{(3)}\right|<\left|b \cos \beta-a_{y}^{(3)}\right|, a_{y}^{(3)} \neq 0\right) \\
\operatorname{sign}\left(-\frac{a_{z}^{(3)}}{b}\right) \cdot \frac{\pi}{2}, & \left(a_{y}^{(3)}=0, a_{z}^{(3)} \neq 0\right) \\
q_{3,0}, & \left(a_{y}^{(3)}=a_{z}^{(3)}=0\right)
\end{array}\right.
$$

where $q_{3,0}$ is the $q_{3}$ at the current moment, $\beta=\arctan \left(-a_{z}^{(3)} / a_{y}^{(3)}\right)$, and $b=R \& \cos q_{2}+R \& \sin q_{2}$. It should be noted that, $q_{2}$ and $q_{3}$ are calculated according the previously obtained \& and However, the difference between $a$ and $R \sqrt{\frac{8}{4}+\%}$ is unavoidable because of calculation error, and this difference will increase the error 
of solving $q_{2}$ and $q_{3}$. For this problem, the following method can be used: when solving $q_{2}$ and $q_{3}$, let

$$
\left\{\begin{array}{l}
\&_{\text {,new }}=\sqrt{k_{q} \&} \\
\&_{\text {,new }}=k_{q} \&
\end{array}\right.
$$

where $k_{q}=a / R \sqrt{\&+1}$. The above revise of \& and will make sure that $R \sqrt{\&_{1, \text { new }}+\sum_{\text {new }}}=a$, so the error of solving $q_{2}$ and $q_{3}$ caused by the magnitude of $a$ will be eliminated.

\section{SIMULATION}

In this section, the correctness of the inverse kinematics algorithm in this paper is verified through an experiment firstly, and then the accuracy of this algorithm is analyzed through several numerical simulations.

\subsection{Simulation 1: Expected Acceleration with Two Non-Zero Components}

The expected acceleration history curve shown in Fig. 5 is considered here, and the inverse kinematics algorithm in this paper is used to calculate kinematic parameters of the GLJ-3R dynamic centrifuge. Then the calculated parameters are used as the control target of the GLJ-3R dynamic centrifuge, so that the expected acceleration environment could be simulated on the centrifuge. The correctness of the algorithm can be verified by comparing the expected acceleration history curve with the acceleration history curve generated by the experiment.

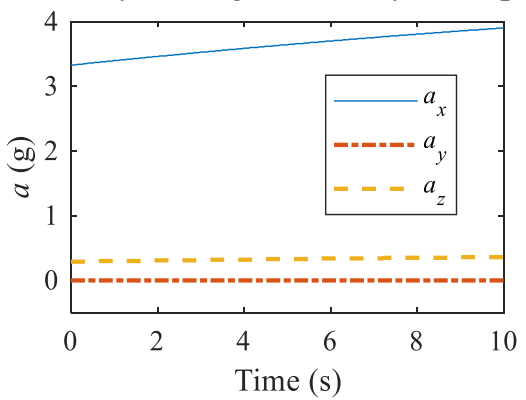

Fig. 5 Expected acceleration history curve

In this simulation, the initial value of $\Delta t$ is chosen as $0.01 \mathrm{~s}$. The kinematic parameters of the centrifuge obtained by the inverse kinematics algorithm of this paper are shown in Fig. 6, where the solid line represents the measured data of the kinematic parameters of the centrifuge, and the dotted line represents the calculation result, which is used as the control target of the centrifuge. It can be seen that the measured data (control result) basically coincides with the target curve (calculation), the angular velocity error is within $0.6 \mathrm{r} / \mathrm{min}$, and the angle control error is within $0.5 \mathrm{deg}$. Since there is no measured data of the angular acceleration of the Axis 1, the calculation results of $\alpha_{1}$ are not given here.

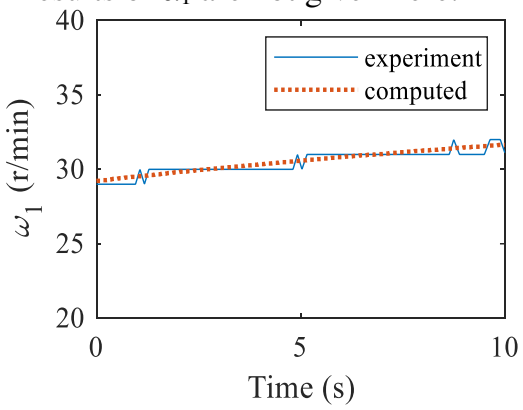

(a) Angular velocity of Axis 1

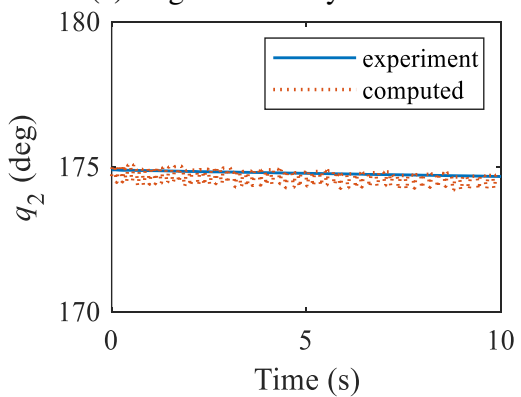

(b) Rotation angle of Axis 2

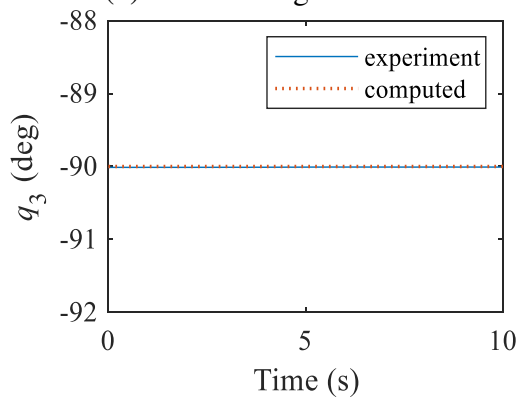

(c) Rotation angle of Axis 3

Fig. 6 Calculated kinematic parameters of the centrifuge

After the centrifuge is controlled according to the calculated $\omega_{1}, q_{2}$ and $q_{3}$, the acceleration at point $O$ on the centrifuge is shown in Fig. 7. The solid line in the figure represents the expected acceleration, and the dotted line represents the measured acceleration. It can be seen that if the inverse kinematics solution in this paper is used as the control target of the dynamic centrifuge, the expected acceleration history curve will be simulated on the centrifuge, and the maximum error is about $0.2 \mathrm{~g}$ ( $\mathrm{g}$ is the acceleration of gravity, taking $9.81 \mathrm{~m} / \mathrm{s}^{2}$ ).

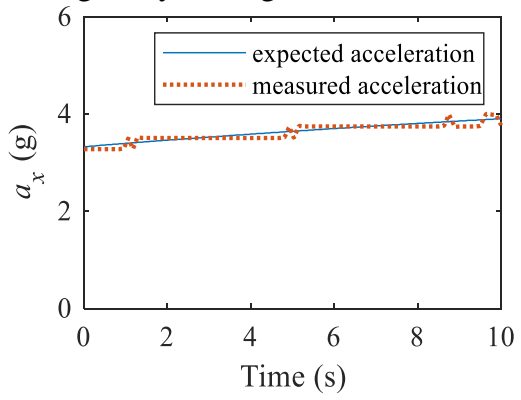

(a) $x$ component 


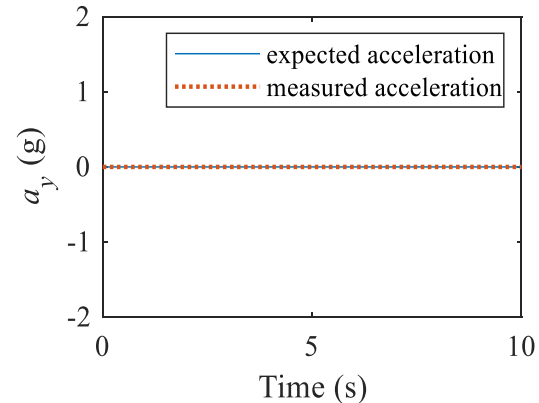

(b) $y$ component

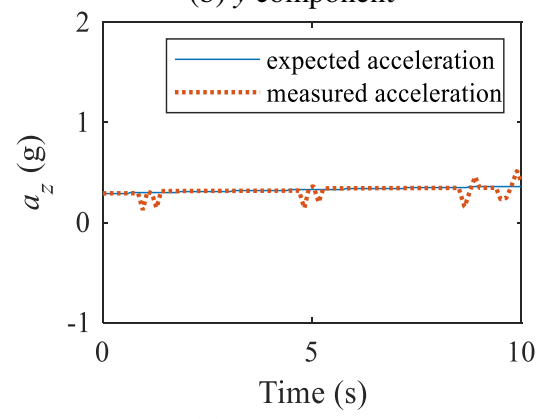

(c) $z$ component

Fig. 7 Expected value and measurement of the acceleration at point $O$ on the centrifuge

The above-mentioned error consists of two parts: 1) the acceleration error of the inverse kinematics solution, this error causes the deviation between the control target and its expected value since the control target is calculated by the inverse kinematics algorithm, 2) the control error, that is, the error caused by the deviation between the control result and the control target. For this article, the main concern is the former error. The error is shown in Fig. 8. It can be seen that the acceleration error of the inverse kinematics solution is less than $0.0004 \mathrm{~g}$, which is much less than the total error of $0.2 \mathrm{~g}$. Therefore, the acceleration error in the experiment mainly comes from the control error.

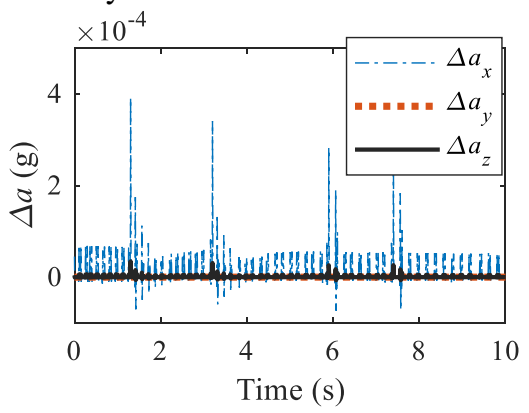

Fig. 8 Acceleration error of the inverse kinematics solution

\subsection{Simulation 2: Expected Trapezoidal Acceleration}

The expected trapezoidal acceleration history curve shown in Fig. 9 is considered in this simulation, and its math expression is

$$
a_{z} / \mathrm{g}= \begin{cases}t, & t \in[0,1] \\ 1, & t \in(1,2] \\ 3-t, & t \in(2,3]\end{cases}
$$

where $g=9.81 \mathrm{~m} / \mathrm{s}^{2}$. In order to use the inverse kinematics algorithm in this paper, firstly, the acceleration curve needs to be divided into non-decreasing segment and non-increasing segment, and then these segments should be solved in forward and reverse directions respectively. The dividing point is the $*$ in Fig. 9. In this simulation, the initial value of $\Delta t$ is chosen as $0.01 \mathrm{~s}$. The kinematic parameters of the centrifuge obtained by the inverse kinematics algorithm is shown in Fig. 10.

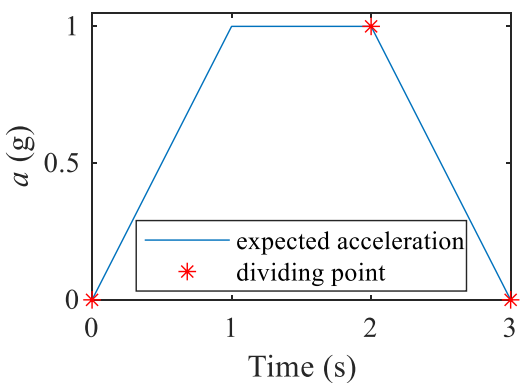

Fig. 9 Expected trapezoidal acceleration history curve

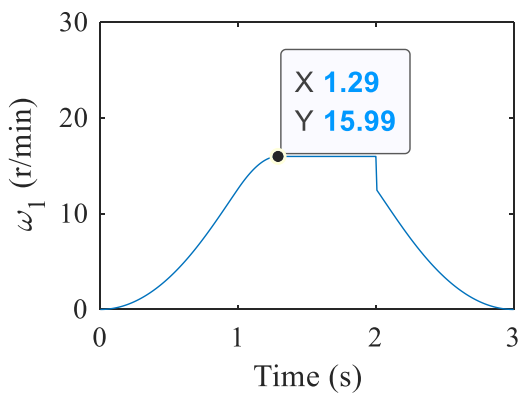

(a) Angular velocity of Axis 1

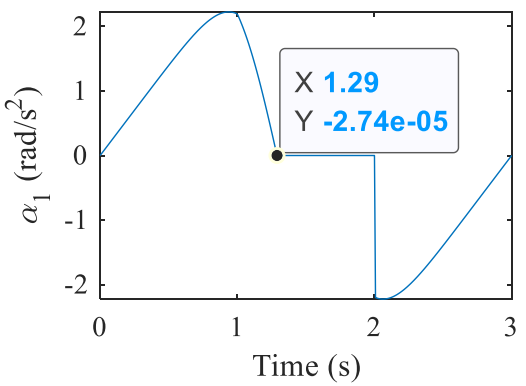

(b) Angular acceleration of Axis 1

Fig. 10 Calculated kinematic parameters (trapezoidal acceleration)

It can be seen that the angular velocity $\omega_{1}$ of the Axis 1 has a sudden change near $t=2 \mathrm{~s}$. In order to analyze the cause of this sudden change, the expected acceleration curve during the timespan of $[0 \mathrm{~s}, 2 \mathrm{~s}]$ is observed firstly. It can be seen that before $t=1 \mathrm{~s}, a$ increases monotonously and then remains at the level of $1 \mathrm{~g}$. Then the angular velocity curve should be checked, it can be seen that $\omega_{1}$ 
has not reached a stable value at $t=1 \mathrm{~s}$. It is easy to know that $\omega_{1}$ will continue to increase when $a$ is rising; so when $a$ just reaches the platform stage, $\omega_{1}$ must be less than its stable value due to the existence of the angular acceleration. Therefore, when $a$ just reaches the platform stage, $\omega_{1}$ will continue to increase, and the angular acceleration $\alpha_{1}$ will gradually reduce to zero from a positive value, and when $\alpha_{1}$ reduce to zero (at about $t=1.29 \mathrm{~s}$ in this simulation) $\omega_{1}$ just reaches its stable value. The result in Fig. 10 also confirms this. Therefore, the reason for this sudden change in the curve of $\omega_{1}$ could be analyzed as follows.

1) When solving the kinematic parameters corresponding to the last segment $(2 \mathrm{~s}<t \leqslant 3 \mathrm{~s})$ of the acceleration curve in the reverse direction, since the dividing point is at $t=2 \mathrm{~s}$, the reverse calculation also ends at $t=2 \mathrm{~s}$, and $\omega_{1}$ has not yet reached the stable value at $t=$ $2^{+} \mathrm{s}$, here $2^{+}$represents a number that is a little more than 2 .

2) However, for the acceleration segment of $0 \mathrm{~s} \leqslant t \leqslant$ $2 \mathrm{~s}$, the calculation direction is forward, and $\omega_{1}$ remains at the stable value in the time interval of $[1.29 \mathrm{~s}, 2 \mathrm{~s}]$, namely $\omega_{1}$ reaches the stable value when $t=2 \mathrm{~s}$.

Therefore, the angular velocity curve is indeed discontinuous at $t=2 \mathrm{~s}$.

The history curve of angular acceleration $\alpha_{1}$ can be derived from the angular velocity curve, and then the history curve of acceleration $a$ at point $O$ on the dynamic centrifuge can be calculated by bringing the obtained $\omega_{1}$, $\alpha_{1}, q_{2}$ and $q_{3}$ into Eq. (2). The acceleration error of the inverse kinematics solution in this paper can be obtained by calculating the difference between the expected acceleration history curve and the calculated acceleration history curve. The error is shown in Fig. 11. Since the $x$ and $y$ components of the acceleration error are much smaller than the $z$ component, only the $z$ component result is given here. It can be seen from the figure that the inverse kinematics solution has a large error at $t=2 \mathrm{~s}$, which is caused by the sudden change of angular velocity. Sudden changes in angular velocity will cause the angular acceleration at $t=2 \mathrm{~s}$ to be very large, thereby introducing an obvious tangential acceleration component, which will eventually cause this large error at $t=2 \mathrm{~s}$.

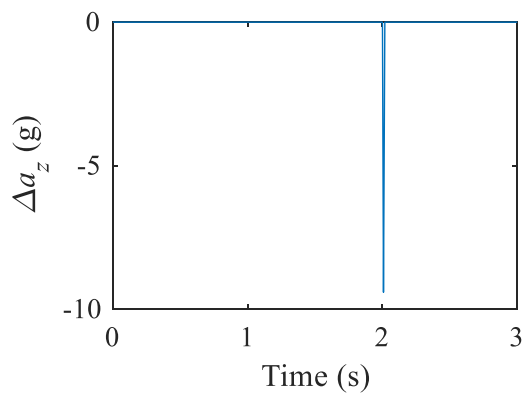

Fig. 11 Acceleration error of the inverse kinematics solution
After the analysis (in the penultimate paragraph above) of the cause of the sudden change of the obtained angular velocity, it can be seen that if the second dividing point of acceleration curve is changed to the midpoint of the overload platform stage, the problem of the sudden change of the obtained angular velocity could be solved. The dividing point after this change is shown in Fig. 12.

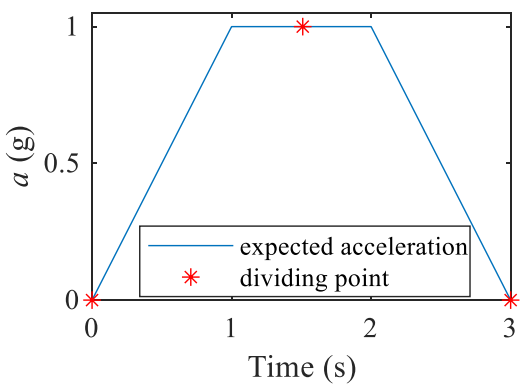

Fig. 12 Dividing points after the change

After the change of the dividing point, the calculated kinematics parameters are shown in Fig. 13, and the corresponding acceleration error of the inverse kinematics solution is shown in Fig. 14. It can be seen from Figs. 13 and 14 that after the dividing point is correctly chosen, the calculated angular velocity is smooth and the acceleration error is greatly reduced.

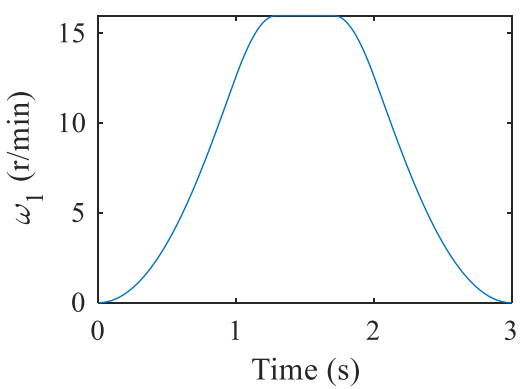

(a) Angular velocity of Axis 1

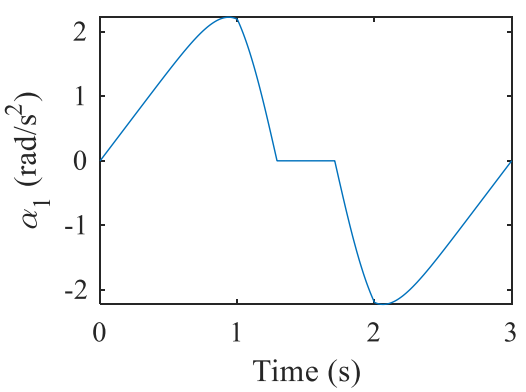

(b) Angular acceleration of Axis 1

Fig. 13 Calculated kinematic parameters (after the change of the dividing points) 


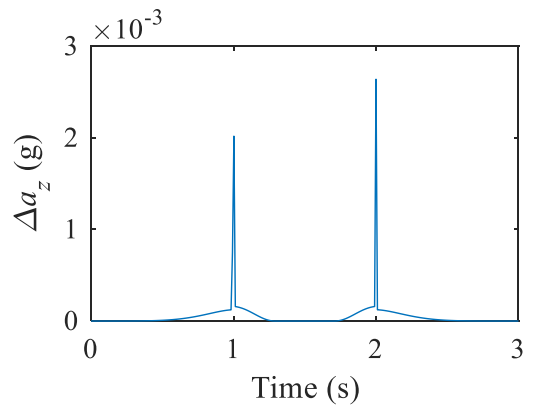

Fig. 14 Acceleration error of the inverse kinematics solution (after the change of the dividing points)

\subsection{Simulation 3: Expected Acceleration from [2]}

The following expected trapezoidal acceleration history curve shown in Fig. 15 is considered in this simulation, and its math expression is expressed in Eq. (11). As shown in Fig. 15, the dividing point are also represented by the mark "*", and here the dividing points at the end of the acceleration platform stage are all avoided.

$$
a_{z} / \mathrm{g}=\left\{\begin{array}{lr}
1, & t \in[0,1] \\
6(t-1)+1, & t \in(1,3] \\
13, & t \in(3,4] \\
13-4(t-4), & t \in(4,7] \\
1, & t \in(7,7.2] \\
8(t-7.2)+1, & t \in(7.2,7.825] \\
6, & t \in(7.825,8.8] \\
6-5(t-8.8), & t \in(8.8,9.8]
\end{array}\right.
$$

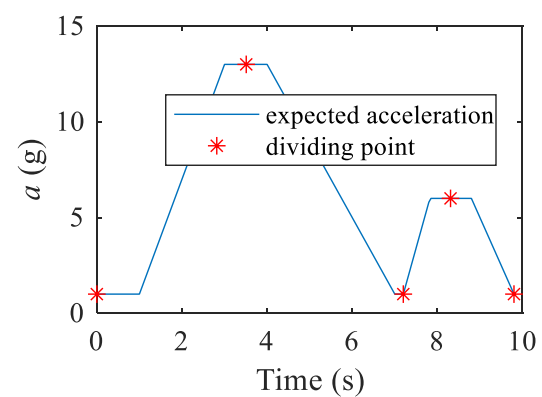

Fig. 15 Expected trapezoidal acceleration history curve in [2]

In this simulation, the initial value of $\Delta t$ is chosen as $0.01 \mathrm{~s}$. The kinematic parameters of the centrifuge obtained by the inverse kinematics algorithm is shown in Fig. 16, and the corresponding acceleration error of the inverse kinematics solution is shown in Fig. 17. Similarly, since the $x$ and $y$ components of the acceleration error are much smaller than the $z$ component, only the $z$ component result is given here. As shown in Fig. 17, if the initial value of $\Delta t$ is chosen as $0.005 \mathrm{~s}$, the corresponding acceleration error could be further reduced to $0.01 \mathrm{~g}$, while in [2], the error is $0.06 \mathrm{~g}$.

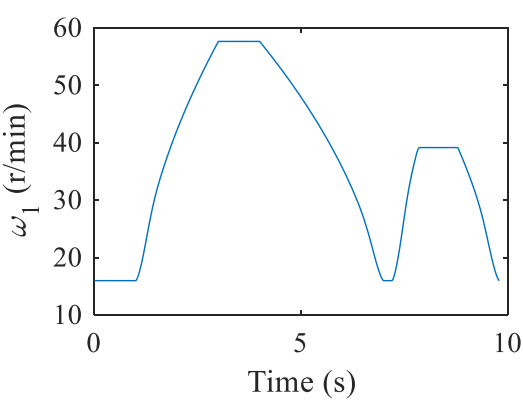

(a) Angular velocity of Axis 1

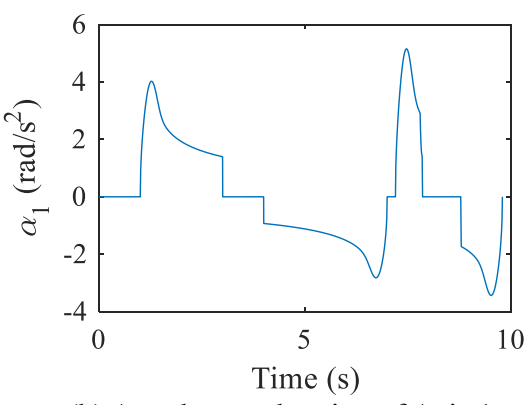

(b) Angular acceleration of Axis 1

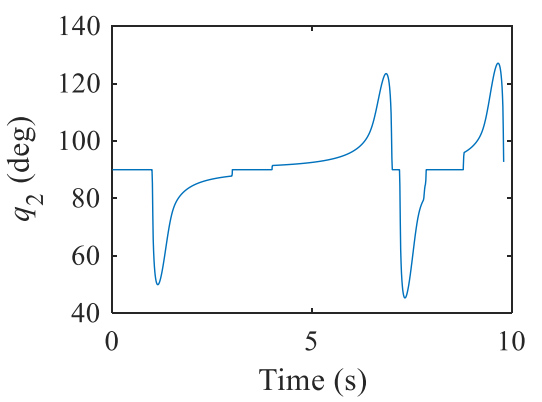

(c) Rotation angle of Axis 2

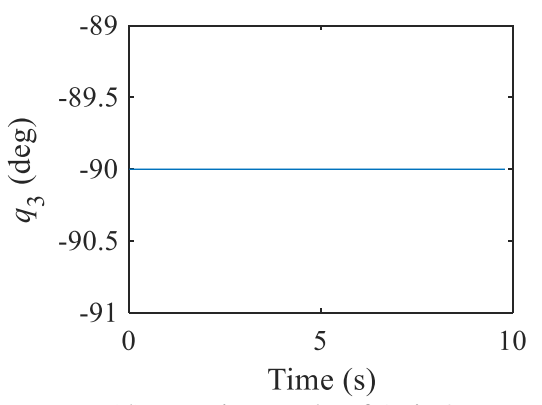

(d) Rotation angle of Axis 3

Fig. 16 calculated kinematic parameters

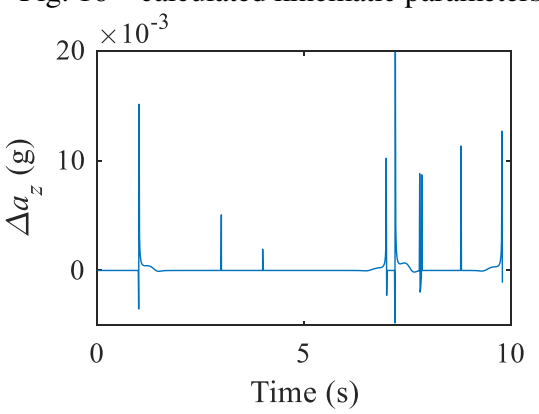

(a) $\Delta t=0.01 \mathrm{~s}$ 


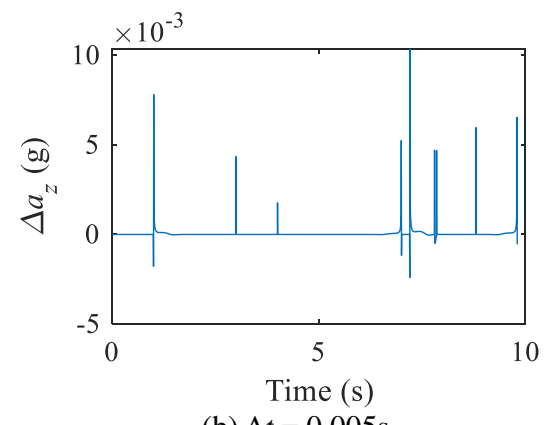

(b) $\Delta t=0.005 \mathrm{~s}$

Fig. 17 Acceleration error of the inverse kinematics solution

The method of adaptively selecting the size of time step has been given in Section 2.2.1. If this method is not available, namely the time step is fixed; the angular acceleration curve corresponding to this example is shown in Fig. 18. It can be seen from the figure that there is high frequency oscillation in the angular acceleration curve. The theory in Section 2.2.1 has predicted this high-frequency oscillation, and the simulation results here verify the theory.

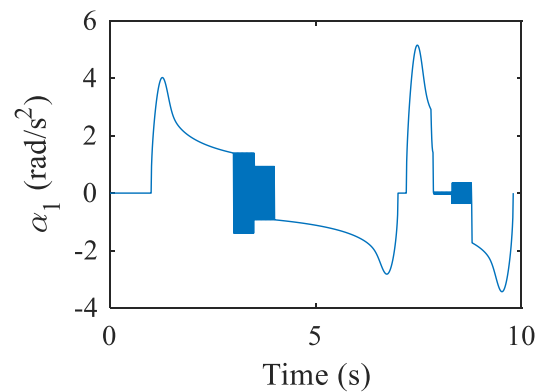

Fig. 18 Angular velocity of Axis 1 (the method of fixed time step)

Simulation 4: Expected Acceleration with Three Non-Zero Components

The following history curve of acceleration with three non-zero components shown in Fig. 19 is considered in this simulation. Its math expression is shown in Eq. (12).

$$
\left\{\begin{array}{l}
a_{x}=10 \mathrm{~g} \sin (t) \\
a_{y}=5 \mathrm{~g} \cos (t) \\
a_{z}=7.5 \mathrm{~g} \cos (2 t)
\end{array}\right.
$$

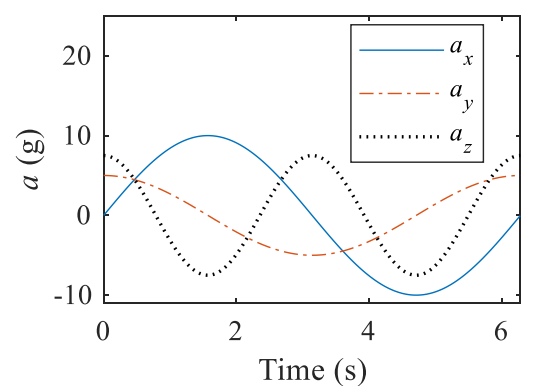

Fig. 19 History curve of expected acceleration with three non-zero components

In this simulation, the initial value of $\Delta t$ is chosen as
$0.01 \mathrm{~s}$. The kinematic parameters of the centrifuge obtained by the inverse kinematics algorithm is shown in Fig. 20, and the corresponding acceleration error of the inverse kinematics solution is shown in Fig. 21. By comparing Figs. 8, 14, 17 and 21, it can be seen that, the error of this algorithm is relatively large (reach $0.02 \mathrm{~g}$ ) at the corner of the acceleration curve. If the acceleration curve is smooth and without corner, the magnitude of the error will be rather small (about $10^{-4} \mathrm{~g}$ ).

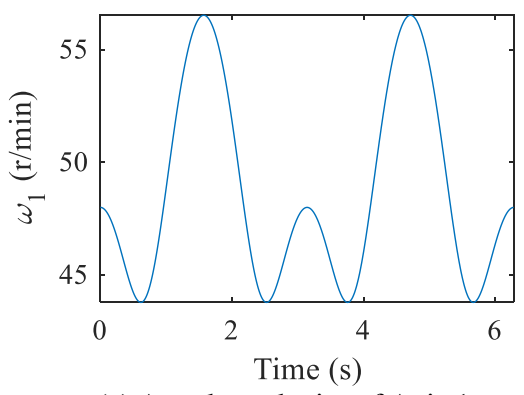

(a) Angular velocity of Axis 1

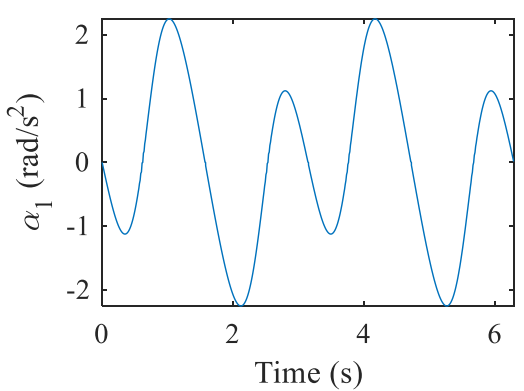

(b) Angular acceleration of Axis 1

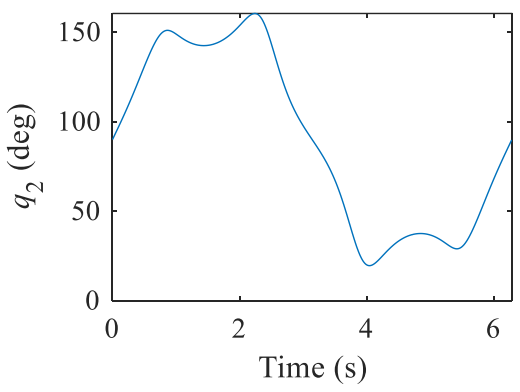

(c) Rotation angle of Axis 2

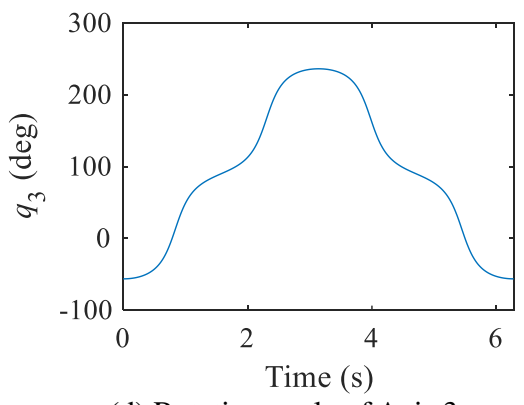

(d) Rotation angle of Axis 3

Fig. 20 Calculated kinematic parameters 


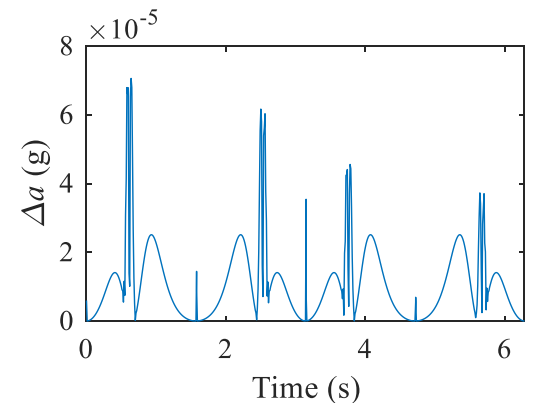

Fig. 21 Acceleration error of the inverse kinematics solution

\section{CONCLUSIONS}

Firstly, the correctness of the inverse kinematics algorithm in this paper is verified through an experiment, and then a series of numerical simulations show that: 1) it is more suitable to choose the dividing point of the acceleration curve at the midpoint of the overload platform stage, instead of at the corner of the acceleration curve; 2 ) the method of adaptively selecting the size of time step in the Section 2.2.1 is effective and avoids the high-frequency oscillation type error of the kinematic parameter solution; 3) the algorithm has a large error at the corner of the acceleration curve, which can be up to $0.02 \mathrm{~g}$, while if the acceleration curve is smooth and has no corner, the magnitude of the error is about $10^{-4} \mathrm{~g}$; 4) this algorithm has strong applicability and is suitable for expected acceleration curves with one, two or three non-zero components, or acceleration curves containing corners; 5) in this paper the algorithm in [2] is improved, and the calculation accuracy is also refined.

\section{DECLARATION}

\section{Acknowledgements}

The authors sincerely thanks to associate Professor Ya-Hong Zhang of Xi'an Jiaotong University for her critical discussion and reading during manuscript preparation.

\section{Funding}

Supported by National Natural Science Foundation of China (Grant No. 12072247)

\section{Availability of data and materials}

The datasets supporting the conclusions of this article are included within the article.

\section{Competing interests}

The authors declare no competing financial interests.

\section{Authors' contributions}

The author' contributions are as follows: Bang-Zhao Zhou was in charge of the whole trial; Shuai Zhang, Min Yang and Sheng-Lai Chen wrote the manuscript; Feng Ou and Ya-Hong Zhang assisted with sampling and laboratory analyses.

\section{References}

[1] Q. Shen, B. Jiang, V. Cocquempot, Fault-Tolerant Control for T-S Fuzzy Systems With Application to Near-Space Hypersonic Vehicle With Actuator Faults, IEEE Transactions on Fuzzy Systems, 20 (2012) 652-665.

[2] M. Yang, Y. Zhang, S. Zhang, G. Dong, X. Zhang, Kinematics Model and Acceleration Simulation Algorithm for a Three-Axis Centrifuge, Journal of Xi'an Jiaotong University, 51 (2017) 159-164.

[3] E.V. Vega, K.Y. Lee, An experimental study on laminar CH4/O2/N2 premixed flames under an electric field, Journal of Mechanical Science and Technology, 22 (2008) 312-319.

[4] Y.-s. CHEN, S. ZHONG, H.-l. GUO, Advances on Dynamic Problems of Hypersonic Space Vehicle, Science Technology and Engineering, 12 (2012) 6401-6409.

[5] Q. Zong, F. Wang, B. Tian, R. Su, Robust adaptive dynamic surface control design for a flexible air-breathing hypersonic vehicle with input constraints and uncertainty, Nonlinear Dynamics, 78 (2014) 289-315.

[6] W. Huang, S. Li, J. Liu, Z. Wang, Investigation on high angle of attack characteristics of hypersonic space vehicle, Science China Technological Sciences, 55 (2012) 1437-1442.

[7] T.R. Kane, R.R. Ryan, A.K. Banerjeer, Dynamics of a cantilever beam attached to a moving base, Journal of Guidance, Control, and Dynamics, 10 (1987) 139-151.

[8] L. Li, D. Zhang, Y. Guo, Dynamic modeling and analysis of a rotating flexible beam with smart ACLD treatment, Composites Part B: Engineering, 131 (2017) 221-236.

[9] X. Liu, W. Liu, Y. Zhao, Unsteady Vibration Aerodynamic Modeling and Evaluation of Dynamic Derivatives Using Computational Fluid Dynamics, Mathematical Problems in Engineering, 2015 (2015) 813462.

[10] Y. Zhang, M. Zhang, S. Zhang, X. Zhang, Experimental Investigation on Bending and Torsional Vibration Control of Shallow Shells, Journal of Experimental Mechanics, 32 (2017) 333-341.

[11] X.-c. Gen, C. Jin, Advances of high $+\mathrm{GZ}$ integrated protection for high performance fighter pilot, CHINESE JOURNAL OF AEROSPACE MEDICINE, 13 (2002) 60-64.

[12] X.-c. Gen, The Research and Application of Aviation Acceleration Physiology, Flight Surgeon, 32 (2004) 189-196.

[13] Y. Zhang, C. Yang, X. Sun, Biomedical problems, developments and application prospects of artificial gravity, Space Medicine \& Medical Engineering, 24 (2011) 423-428.

[14] L.L. Dong, G.R. Run, H.J. Liao, THE DYNAMIC CENTRIFUGE MODEL TEST IN GEOTECHNICAL ENGINEERING, Chinese Journal of Rock Mechanics and Engineering, 19 (2000) 789-793.

[15] W. Pan, L. Wang, Principles of G-load Simulation for a Novel Sustained-G Flight Simulator, ACTA AERONAUTICA ET ASTRONAUTICA SINICA, 31 (2010) 2159-2165. 
[16] Y. You, C. Wu, J.-s. You, X.-y. Xu, Y.-y. You, Study of Motion System of Flight Simulator of Persisted Load, JOURNAL OF SYSTEM SIMULATION, 19 (2007) 1154-1156.

[17] H.-y. Xu, L.-w. Guan, L.-p. Wang, X. Chen, Topology Optimization for the Arm of Flight Simulator under Inertial Loads, Journal of Mechanical Engineering, 50 (2014) 14-23.

[18] H. Liu, L.W. Guan, Dynamic Modeling of a High-Dynamic Flight Simulator, Applied Mechanics and Materials, 687-691 (2014) 610-615.

[19] H.Y. Xu, L.W. Guan, L.P. Wang, The Statics and Dynamics Analysis of High-Dynamic Flight Simulator Based on Substructure Method, Advanced Materials Research, 658 (2013) 327-334.

[20] Y. Zhang, S. Zhang, S. Chen, X. Zhang, General Kinematics Model and Coupling Movement Analysis for a Three-axis Centrifuge Motion Simulator, Journal of Mechanical Engineering, 52 (2016) 135-140.

[21] L. Chang, Z. Liu, N. Wen, S. Wu, Configuration and Mathematical Modeling for Advanced Three-Axis Centrifuge System, Journal of Beijing University of Aeronautics and Astronautics, 41 (2015)
283-288.

[22] D.-f. Zhang, F. Ou, Simulation Test Principle for a Type of Aircrafts with Variable Accelerations, Equipment Environmental Engineering, (2015) 56-60.

[23] R. Crosbie, D. Kiefer, Controlling the Human Centrifuge as A Force and Motion Platform for the Dynamic Flight Simulation, in: Flight Simulation Technologies Conference, 1985.

[24] J. Vidakovic, G. Ferenc, M. Lutovac, V. Kvrgic, Development and implementation of an algorithm for calculating angular velocity of main arm of human centrifuge, in: 2012 15th International Power Electronics and Motion Control Conference (EPE/PEMC), 2012, pp. DS2a.17-11-DS12a.17-16.

[25] L. Guan, H. Liu, M. Fu, Real-time Motion Planning Algorithm for Dynamic Flight Simulators, Journal of Tsinghua University (Science and Technology), 55 (2015) 709-715. 